\title{
Suministro y demanda de sangre durante la pandemia de COVID-19. Una revisión
}

Rita C. Gutiérrez-Hernández ${ }^{1 *}$ y Jesús Madrigal-Anaya²

${ }^{1}$ Departamento de Banco de Sangre, Centro Médico Nacional La Raza, Instituto Mexicano del Seguro Social; '2Departamento de Toxicología, Clínica Hospital Juárez de México. Ciudad de México, México

\section{Resumen}

La propagación tan rápida del nuevo coronavirus 2 del síndrome respiratorio agudo grave (SARS-CoV-2), que se informó por primera vez en Wuhan (China) a finales de diciembre de 2019, y produce la enfermedad caracterizada por neumonía llamada enfermedad por coronavirus 2019 (COVID-19), ha causado un impacto significativo en los sistemas de salud en todo el mundo y la interrupción de la vida cotidiana. A finales de junio el número de casos confirmados es de 9,188,362 millones, con más de 474,339 muertes, y aproximadamente 4,937,282 personas recuperadas. Las estrictas medidas para restricción de la movilidad y medidas de distanciamiento social impuestas por los gobiernos, más el miedo y pánico resultado de manera abrumadora entre el público general, ha comprometido de manera importante la sostenibilidad del suministro de sangre segura, el cual se vuelve particularmente desafiante. La demanda de sangre y productos sanguíneos puede disminuir durante una pandemia debido al aplazamiento de cirugías electivas. Medidas como el distanciamiento físico y el bloqueo total de las ciudades, las provincias o países que intentan frenar la propagación de la infección pueden provocar una disminución importante en el suministro y una escasez general de productos sanguíneos. Los hospitales deben contar con un plan de manejo de sangre para emergencias en la planificación de preparación sobre la sostenibilidad y seguridad del suministro de sangre. Esta revisión considera el impacto de la pandemia de COVID-19 en el suministro de sangre y especifica aspectos importantes del manejo de los donantes, y la utilización apropiada de los componentes sanguíneos.

PALABRAS CLAVE: Suministro. Impacto. Demanda. Componentes sanguíneos. COVID-19. SARS-CoV-2.

\section{Blood supply and demand during the COVID-19 pandemic. A review}

\begin{abstract}
The rapid spread of the new severe acute respiratory syndrome coronavirus 2 (SARS-CoV-2), which was first reported in Wuhan (China) in late December 2019, and produces the disease characterized by pneumonia called coronavirus disease 2019 (COVID-19), has caused a significant impact on health systems worldwide and disruption of daily life; at the end of June the number of confirmed cases is 9,188,362 million with more than 474,339 deaths, and approximately 4,937,282 people recovered. Strict measures to restrict mobility, social distancing measures imposed by governments, plus overwhelming fear and panic among the general public, have significantly compromised the sustainability of the safe blood supply, it becomes particularly challenging. The demand for blood and blood products may decrease during a pandemic due to the postponement of elective surgeries, measures such as physical distancing and the total blockade of cities, provinces or countries that try to stop the spread of the infection can cause a significant decrease in the supply and a general shortage of blood products. Hospitals must have a blood management plan for emergencies in preparedness planning on the sustainability and safety of the blood supply. This review considers the impact of the COVID-19 pandemic on blood supply and specifies important aspects of donor management, and the proper use of blood components.
\end{abstract}

KEY WORDS: Supply. Impact. Demand. Blood components. COVID-19. SARS-CoV-2.

Correspondencia:

${ }^{*}$ Rita C. Gutiérrez-Hernández

E-mail: rit_gu@yahoo.com.mx

0016-3813/@ 2021 Academia Nacional de Medicina de México, A.C. Publicado por Permanyer. Este es un artículo open access bajo la licencia
Fecha de recepción: 09-09-2020

Fecha de aceptación: 09-02-2021
Gac Med Mex. 2021;157(Supl 3):S55-S67

Disponible en PubMed

www.gacetamedicademexico.com

CC BY-NC-ND (http://creativecommons.org/licenses/by-nc-nd/4.0/). 


\section{Introducción}

La propagación tan rápida del nuevo coronavirus 2 del síndrome respiratorio agudo grave (SARS-CoV-2), que se informó por primera vez en Wuhan, China, a finales de diciembre 2019, y produce la enfermedad caracterizada por neumonía llamada enfermedad por coronavirus 2019 (COVID-19), ha causado un impacto significativo en los sistemas de salud en todo el mundo y la interrupción de la vida cotidiana. A finales de junio el número de casos confirmados es de $9,188,362$ millones, con más de 474,339 muertes, y aproximadamente 4,937,282 personas recuperadas'.

EI SARS-CoV-2 presenta un periodo de incubación de 1-14 días generalmente (promedio de 5-6 días, el más largo reportado en la literatura es de 24 días) ${ }^{2}$. Causa infección asintomática en una importante cantidad de individuos (30\%). Esto genera importantes desafíos en el reclutamiento de donantes de sangre, la recolección y la seguridad de esta. En todo el mundo el número de donantes se redujo de manera considerable, incluyendo los países donde la captación de sangre es de manera voluntaria, lo que ha puesto en peligro el stock y suministro de sangre necesario para la transfusión clínica.

Esto como resultado de las medidas estrictamente tomadas para reducción de la movilidad de las personas, la que a nivel internacional ha alcanzado mínimos históricos. Los gobiernos locales han girado órdenes con instrucciones para tomar medidas proactivas con la finalidad de garantizar los suministros de sangre necesarios para su uso clínico; México no ha sido la excepción ante tal situación, considerando que la captación de sangre se obtiene en su mayoría por reposición o familiar aproximadamente en un $98 \%$.

Es importante desarrollar mejores prácticas, garantizar el suministro y la seguridad transfusional. Es el momento de comprender las acciones emprendidas por algunos países durante la pandemia y prepararse para futuras pandemias o cualquier otra contingencia; para la difusión de diferentes políticas y procedimientos que faciliten la provisión oportuna de productos sanguíneos a nuestros pacientes mientras se mantiene un nivel de seguridad en todos los involucrados en la cadena transfusional.

\section{Requerimientos transfusionales de los pacientes con COVID-19}

Las estrictas medidas para restricción de la movilidad, medidas de distanciamiento social impuestas por los gobiernos, más el miedo y pánico resultado de manera abrumadora entre el público general, ha comprometido de manera importante la sostenibilidad del suministro de sangre segura, que se vuelve particularmente desafiante.

El Centro Nacional de Enfermedades Infecciosas de Singapur realizó una evaluación sobre las directrices utilizadas sobre el suministro de sangre en respuesta a la pandemia de COVID-19 considerando los hospitales Renci Community Hospital y Tan Tock Seng Hospital (1,700 camas para cuidados agudos): de 572 pacientes con COVID-19, 19 requirieron atención en unidad de terapia intensiva (UTI) y 9 de los 572 requirieron transfusiones, 5 hombres y 4 mujeres, la media para la edad fue 70 años. Ninguno presentó evidencia clínica de hemólisis.

De los pacientes transfundidos uno presentaba síndrome de dificultad respiratoria (SDRA), infarto de miocardio sin elevación del segmento ST, fibrilación auricular, accidente cerebrovascular cardioembólico y lesión renal aguda (IRA), que requirió terapia de reemplazo renal continuo (CRRT), sin evidencia de sangrado; se transfundieron concentrados de eritrocitos (CE) por hemoglobina $(\mathrm{Hb})$ baja de $8.1 \mathrm{mg} / \mathrm{dl}$. Otro paciente de los transfundidos presentaba SDRA, shock séptico, IRA con CRRT e insuficiencia multiorgánica y presentó sangrado de tubo digestivo; presentaba pancitopenia, por lo que se realizó examen de médula ósea, que mostró hematopoyesis trilinaje adecuada; presentaba anemia atribuida a causa multifactorial y también a la respuesta inflamatoria; por el sangrado de tubo digestivo, se transfundieron 24 unidades de CE y un pool de plaquetas.

Un paciente más con asma, hipertensión, hiperlipidemia y cardiopatía isquémica, con SDRA, IRA y CRRT más oxigenación por membrana extracorpórea, presentaba sangrado importante, se transfundió plasma fresco congelado (PFC) y pool de plaquetas; el paciente falleció por fracaso multiorgánico. Otro paciente transfundido con hipertensión, ataque isquémico transitorio y SDRA, que presentó infarto de miocardio, fue sometido a derivación de arteria coronaria, se transfundieron agentes antiplaquetarios y heparina de bajo peso molecular, y se transfundieron dos unidades de CE. Un paciente con hipertensión, hiperlipidemia, cardiopatía isquémica y gastritis y SDRA, así como IRA más CRRT, evacuaciones melénicas, presentó necrosis en miembro pélvico izquierdo por trombosis venosa profunda, requirió nueve unidades de CE y falleció por falla multiorgánica. 
La siguiente paciente, complicada con SDRA, lesión hepática y renal aguda. Presenta anemia atribuida a infección severa y el sangrado en sitio de línea central, y ameritó dos unidades de CE.

Otro de los pacientes con SDRA, complicado con trombosis venosa profunda de miembro superior derecho secundaria a una colocación de línea venosa central, anticoagulado, desarrolló rectorragia, presentaba infección dual con citomegalovirus (CMV) y proctitis, por lo que se transfundieron CE.

Dos pacientes más presentaban premenopausia y anemia por deficiencia de hierro, con síntomas de anemia exacerbados por la neumonía y recibieron un CE cada uno y se inició reemplazo con hierro.

Como podemos observar en está serie de casos, los pacientes presentan COVID-19 grave, pero no es la causa aparente de las transfusiones, estas son ocasionadas por comorbilidades que los pacientes presentan; la mayoría de los pacientes con COVID-19 leve no requieren transfusiones, en este estudio solo el $0.36 \%$ de los pacientes que no se encontraban en UTI requirieron transfusión de CE. Sin embargo, el $36.8 \%$ de los pacientes que se encontraban en UTI requirieron transfusión de CE y en menor cantidad PFC, así como la transfusión de plaquetas.

Tres de cada siete pacientes de la UTI presentaron hemorragia gastrointestinal grave y se les transfundieron $\mathrm{CE}^{3}$. Importante tomar en cuenta que Xiao, et al. demostraron la transmisión fecal-oral del SARSCoV-2 como una vía adicional de transmisión por medio de la entrada a las células epiteliales gastrointestinales mediante la enzima convertidora de angiotensina 2. Demostraron el daño a la mucosa del esófago con tinción positiva para SARS-CoV-2 en tejidos obtenidos del esófago, duodeno y recto. Esto es una posibilidad que explica por qué pacientes más gravemente enfermos con COVID-19 presentan daño a la mucosa gastrointestinal por infección generada por SARS-CoV-2 o también gastritis por estrés/urémica o por infección oportunista como CMV, causa colitis y predispone a un mayor requerimiento de transfusión de CE en la fase crítica ${ }^{4}$.

Es necesario considerar que en los pacientes con antecedentes de anemia por deficiencia de hierro con síntomas exacerbados por esta es primordial evaluar el riesgo de complicaciones de la transfusión. Bien iniciar terapia de reemplazo con hierro con el objetivo de mejorar la eritropoyesis, es mejor manejar de manera prudente el uso de transfusiones basados en las guías de manejo de transfusiones.
El uso de plasma fresco y plaquetas se transfunden mínimamente, principalmente los que presentan valores aumentados de dímero $\mathrm{D}$, sin embargo, este parámetro no se considera específico y se reconoce que se eleva en pacientes hospitalizados con inflamación severa y enfermedad hepática; ninguno de los pacientes presentados tenía criterios para coagulación intravascular diseminada y tampoco criterios para transfusión de crioprecipitados y fibrinógeno. La transfusión de plaquetas se usó para disminuir el riesgo de sangrado durante los procedimientos invasivos.

Estos pacientes no requirieron productos sanguíneos industrializados como: concentrado de factor VIII, concentrados de complejo de protrombina, factor IV o factor VIII recombinante.

Durante los meses de febrero a marzo del 2020 hubo una reducción importante de las transfusiones en el Health City en comparación con los 12 meses anteriores, con una media de 1,270 unidades de CE por mes en el 2019 en comparación con 1,063 unidades de CE por mes de febrero a marzo del 2020 (reducción del 16\%). También hubo una reducción en PFC de 193 a 245 unidades por mes, disminución del $21.2 \%$ y componentes plaquetarios de 166 a 197 unidades por mes equivalente a una disminución del 17.7\%. Si bien el hospital Tock Seng aún admite pacientes no COVID-19, esta reducción en las transfusiones es por el aplazamiento de las cirugías electivas y la importante disminución en la ocupación de camas para hospitalizaciones durante febrero a marzo de 2020.

Esto también se observó de marzo a mayo de 2003 con la epidemia de SARS, donde el Hospital Tan Tock Seng era designado para manejar solo pacientes con sospecha o confirmados con SARS; los requerimientos de transfusión de sangre durante ese periodo mostraron una disminución del $66.5 \%$ de un promedio de 941 unidades mensuales de CE transfundidos en 2002 a 315 unidades mensuales transfundidas, y una disminución del uso de PFC de 500 unidades y 289 para concentrados plaquetarios en 2002 a 211 unidades de PFC disminución del $57.8 \%$ y 155 de concentrados plaquetarios, igual a una disminución del $46.3 \% \%^{5}$.

Los desafíos que enfrentan los bancos de sangre durante una pandemia infecciosa son asegurar y proteger el suministro de sangre ${ }^{6}$. Si bien la demanda de sangre y productos sanguíneos puede disminuir durante una pandemia debido al aplazamiento de cirugías electivas, medidas como el distanciamiento 
físico y el bloqueo total de las ciudades, las provincias o países que intentan frenar la propagación de la infección pueden provocar una disminución importante en el suministro y una escasez general de productos sanguíneos. Esto se observó en abril a julio de 2003 en Bejing (China) durante el brote de SARS, donde fue necesario importar sangre y productos sanguíneos de otras provincias chinas para garantizar la disponibilidad para uso clínico en pacientes ${ }^{7}$.

Los hospitales deben contar con un plan de manejo de sangre para emergencias en la planificación de preparación sobre la sostenibilidad y seguridad del suministro de sangre. Los primeros informes han descrito anemia leve en COVID-19 pacientes ingresados en la $\mathrm{UCl}$. En general, la mayoría de las etiologías comunes de la anemia en la $\mathrm{UCl}$ son inflamación y deficiencia de hierro. Como en pacientes quirúrgicos, hierro, vitamina B12, ácido fólico y eritropoyetina pueden administrarse en pacientes críticamente enfermos. Las formulaciones de hierro intravenoso son superiores a las formulaciones orales en tales pacientes, ya que la captación de hierro enteral se reduce en estados inflamatorios debido al aumento de la expresión de hepcidina ${ }^{8}$.

Por lo tanto es importante considerar cuidadosamente cada indicación de transfusión, teniendo en cuenta factores individuales como la edad, el estado del volumen del paciente y las enfermedades concomitantes; en estos pacientes es importante antes de la transfusión de CE alogénicos utilizar medidas que aumenten el suministro de oxígeno y la saturación de este, así como el gasto cardiaco. El volumen total de eritrocitos no siempre se refleja en los valores de $\mathrm{Hb}$ y es incorrecto centrarse simplemente en estos valores para indicar la transfusión.

Es necesario seguir las guías internacionales para la transfusión, limitar el volumen transfundido y evaluar siempre con pruebas de rastreo de anticuerpos irregulares cuando los pacientes han recibido múltiples transfusiones y de diversos donantes, con la finalidad de detectar alosensibilización que puede desencadenar una reacción transfusional fatal en próximas transfusiones; excepto cuando los pacientes presenten sangrado masivo activo.

\section{Impacto de la pandemia de COVID-en el suministro de sangre}

Conforme la COVID-19 aumenta sus cifras en el mundo, la prioridad número uno es optimizar el resultado del paciente durante esta catastrófica crisis de atención médica. Los principios del manejo de la sangre del paciente son estrategias fundamentales para mejorar los resultados del paciente y se les debe dar prioridad en esta situación de crisis.

Uno de los objetivos de esta revisión es proporcionar a los médicos y autoridades de atención médica información sobre cómo aplicar los principios del manejo de la sangre durante la pandemia de COVID-19.

Esta revisión considera el impacto de la pandemia de COVID-19 en el suministro de sangre y especifica aspectos importantes del manejo de los donantes, y la utilización apropiada de los componentes sanguíneos.

Es importante evaluar las cuestiones relacionadas con diagnósticos y tratamientos de la anemia en pacientes que requieren cirugía electiva o de emergencia. Es necesario reconocer las consideraciones especiales relacionadas con la oferta-demanda y problemas de costo-beneficio en el manejo de la sangre durante la pandemia de COVID-19, que en la mayoría de los países del mundo ha generado una emergencia sanitaria y socioeconómica sin precedente alguno.

A consecuencia de las restricciones sociales realizadas por los gobiernos para disminuir la propagación del SARS-CoV-2, el número de donaciones de sangre ha disminuido significativamente durante las últimas semanas y meses. A la fecha solo se ha identificado un reporte de caso como evidencia científica documentada de infección por SARS-CoV-2 de un donante de plaquetas, razón por la que se ha producido una preocupación sobre la seguridad de las donaciones de sangre ${ }^{9}$. Se han notificado varios casos en los que se pudo detectar ARN viral específico en el suero de pacientes con COVID-19; estos hallazgos sugieren que la donación de sangre puede ser una ruta de transmisión inexplorada. Sin embargo, la Asociación Estadounidense de Bancos de Sangre (ahora AABB) y los Centros para el Control y la Prevención de Enfermedades (CDC) no han recomendado acciones específicas relacionadas con el SARS-CoV-2 en los centros de recogida de sangre en este momento. El caso reportado en la literatura es de un paciente de sexo masculino de 21 años con anemia aplásica severa que recibió aféresis de plaquetas de una persona que posteriormente fue diagnosticada con COVID-19. El paciente resultó negativo para COVID19 y en espera de trasplante alogénico de células progenitoras hematopoyéticas, al momento de su ingreso sus signos vitales eran estables, no había fiebre; su estado general era bueno, sin evidencias 
de infección, clasificado de acuerdo con su estado por el ECOG (Eastern Cooperative Oncology Group) estadio 1. La biometría hemática con recuento de leucocitos de $270 / \mathrm{mm}^{3}$ (recuentos absolutos de neutrófilos de $\left.30 / \mathrm{mm}^{3}\right), \mathrm{Hb}$ de $8.1 \mathrm{~g} / \mathrm{dl}$ y plaquetas de $7,000 / \mathrm{mm}^{3}$. Inmediatamente después de la admisión fue transfundido con aféresis de plaquetas de un donante aparentemente sano. Un día después de la transfusión de plaquetas, el banco de sangre notificó que el donante de plaquetas fue diagnosticado posteriormente con COVID-19. Los productos sanguíneos se recolectaron dos días antes de que se realizara la transfusión de plaquetas; se consideraba que el donante estaba sano y no tenía fiebre ni síntomas respiratorios en ese momento.

Al receptor se realizó prueba para SARS-CoV-2 usando reacción en cadena de la polimerasa-transcriptasa inversa, el resultado fue negativo. El paciente no mostró ningún síntoma de infección, y no hubo evidencia de neumonía en la tomografía de tórax. Se realizaron tres pruebas más para el SARS-CoV-2, todos los resultados fueron negativos. El paciente se ha mantenido estable y ahora está preparado para el trasplante alogénico programado de células madre hematopoyéticas ${ }^{9}$.

El Centro Europeo para la Prevención y Control de Enfermedades (ECDC) y los CDC sugieren un aplazamiento precautorio de la donación de sangre durante 21 días después de cualquier posible exposición a pacientes confirmados. Además, recomiendan para aquellos que se recuperan de la COVID-19 que deben evitar donar sangre durante al menos 28 días después de la resolución de los síntomas y la finalización de la terapia ${ }^{10}$.

Siguiendo las indicaciones del ECDC con respecto al suministro de sangre ${ }^{10}$, la mayoría de las autoridades nacionales competentes de salud de Europa han emitido algunas recomendaciones sobre la prevención de la transmisión de la infección por SARSCoV-2 por medio de transfusión de componentes sanguíneos. Además de la recomendación de emplear los programas de reservas de sangre basados en el manejo de la sangre del paciente en los hospitales con base en el patient blood management y también se han implementado varias acciones en el marco de la selección de donantes de sangre.

En algunos países europeos la disminución inicial se reporta en aproximadamente el $5-10 \%$ en la recolección de sangre total, pero la mayoría de estos países han mantenido estables sus volúmenes de donaciones ${ }^{11}$.

\section{Efecto de la pandemia de COVID-sobre la prevalencia de anemia y deficiencias hematínicas en pacientes quirúrgicos}

En la cirugía mayor electiva, los pacientes pueden estar expuestos a los efectos de la anemia preoperatoria, la pérdida de sangre y requerir la transfusión de glóbulos rojos, que influye negativamente en el resultado postoperatorio ${ }^{12,13}$.

En pacientes sometidos a procedimientos quirúrgicos mayores, se recomienda que la $\mathrm{Hb}$ defina la anemia preoperatoria $(\mathrm{Hb}<13.0 \mathrm{~g} / \mathrm{ml}$ independientemente del sexo $)^{14,15}$. Considerando esta definición, en un estudio de cohorte con procedimientos electivos la prevalencia general de anemia preoperatoria fue del $36 \%$, más del $70 \%$ de los pacientes con anemia presentaron deficiencia de hierro absoluta o funcional, lo que resultó en eritropoyesis ineficaz ${ }^{16}$. Además, la deficiencia de hierro preoperatoria en pacientes que se someten a cirugía cardiaca está asociada con una mortalidad tres veces mayor a los 90 días $^{17}$. Las deficiencias hematínicas no anémicas también son frecuentes y pueden dificultar la optimización preoperatoria de la $\mathrm{Hb}$ y/o la recuperación de la anemia postoperatoria ${ }^{16}$.

En cuanto a los procedimientos no electivos, hasta el $75 \%$ de los pacientes sometidos a cirugía de reparación de fractura de cadera presentaron $\mathrm{Hb}<13.0 \mathrm{~g} /$ dl al ingreso ${ }^{18}$. Se puede esperar que estas cifras aumenten durante la pandemia de COVID-19 debido a los cambios en la dieta y estilo de vida, agravados por repercusiones en la economía con menores ingresos y poder adquisitivo; las posibles consecuencias es probable que tengan que ver con la reducción del consumo de alimentos frescos, frutas, pescado, carne y productos lácteos (fuente importante de hierro, vitaminas B6, B12, C, ácido fólico y vitamina D). Unido a ello la falta de exposición a la luz solar y atrofia muscular, resultante de restricciones por toque de queda y distanciamiento social. Todo ello generando importantes consecuencias en el metabolismo del hierro y síntesis de $\mathrm{Hb}$, alterando la proliferación de progenitores de glóbulos rojos; también el rendimiento físico y mental podría verse afectado ${ }^{19,20}$. Según el estado de salud general y la duración de la pandemia de COVID-19, es probable que los ancianos sean los más afectados ${ }^{21}$, tomando en cuenta que esta población es la más vulnerable por las importantes comorbilidades que presentan y representan la mayor proporción de pacientes médicos y quirúrgicos hospitalizados (Tabla 1). 
Gaceta Médica de México. 2021;157(Supl 3)

Tabla 1. Recomendaciones del Patient Blood Management (PBM) en pandemia

PBM para el manejo de la sangre de pacientes (del grupo de trabajo IFPBM-SABM)

\begin{tabular}{l|l|l|l}
\hline Herramientas & Deficiencia de hierro y anemia & Pérdida sanguínea y sangrado & Coagulopatia
\end{tabular}

1. Metodología de - Cambiar la cultura en toda su institución

implementación - Difundir las pautas y recomendaciones de manejo de sangre del paciente basadas en evidencia (PBM), detectar y de programa desalentar las prácticas de no evidencia

- Traducir las pautas/recomendaciones basadas en evidencia en la práctica clínica

- Identificar áreas de práctica que necesitan mejoras

2. Dispositivos

- Point-of-care en analizadores de

diagnósticos hemoglobina

- Point-of-care en pruebas para deficiencia de hierro

- Pruebas point-of-care en coagulación y función plaquetaria y terapia dirigida a la meta

- Pruebas diagnósticas rápidas con la presencia de ACOD

3. Dispositivos terapéuticos

4. Productos farmacéuticos

5. Vigilancia con interacciones nutricionales

\section{Principios} generales $(A)$

\section{Identificar y gestionar terapias}

farmacológicas y/o nutricionales que:

- Puede contribuir a la anemia y las deficiencias hematínicas (p. ej., IBP)

- Puede aumentar la absorción de hierro (p. ej., ácido ascórbico)

- Puede perjudicar la absorción ( $p$. ej., algunos suplementos vitamínicos y herbales, té, café o productos lácteos)
- Recuperación celular perioperatoria y postoperatoria (recuperador de células) - HNA

- Antifibrinolíticos (ácido tranexámico, ácido aminocaproico)

- Agentes hemostásicos tópicos

- Agentes vasoconstrictores locales

- Agentes estimulantes de leucocitos y plaquetas, cuando sea apropiado

- Considere una $\mathrm{FiO}_{2}$ alta (100 \%) en pacientes con anemia potencialmente fatal

Identificar y gestionar terapias farmacológicas y/o nutricionales que aumentan el riesgo de sangrado, por ejemplo:

- AINE (incluidos los inhibidores de COX2), antidepresivos, estatinas, antiarrítmicos

- Suplementos de vitaminas y hierbas que incluyen vitamina $\mathrm{E}$, vitamina $\mathrm{K}$, ajo, jengibre, Ginkgo biloba, aceite de pescado, manzanilla, raíz de diente de león, etc.

Identificar, evaluar, controlar la anemia y - Meticulosa cirugía hemostásica la deficiencia de hierro:

- Técnica quirúrgica óptima

- Posicionamiento del paciente

- Evaluar y controlar los trastornos subyacentes que causan anemia y deficiencia de hierro

- Conozca los fármacos asociados con los trastornos de los eritrocitos

- Programa de manejo de la anemia para pacientes prehospitalarios, hospitalarios y posteriores al alta

- Centrarse en pacientes con comorbilidades (diabetes, enfermedad renal crónica e insuficiencia cardiaca congestiva)
- Esfuerzos para detener el sangrado inmediatamente

Técnicas quirúrgicas mínimamente invasivas. Hipotensión permisiva o controlada y administración de líquidos balanceada hasta control del sangrado Lograr euvolemia una vez controlado el sangrado

Cuidado de la presión arterial y el manejo de líquidos, prevenir la hipotermia, hipotensión y acidosis - Minimizar la pérdida iatrogénica de sangre, minimizar cantidad de extracciones de sangre y volumen, minimizar el volumen de sangre desperdiciada (microtainers [tubo para sangre capilar]/pequeños tubos de flebotomía)
- Point-of-care en pruebas de coagulación y de función plaquetaria y terapia dirigida a metas u objetivos

- Pruebas de diagnóstico rápido para detectar la presencia de ACOD si están disponibles

- Concentrado de fibrinógeno

- CCP

- Otros factores de coagulación

- Vitamina K por vía intravenosa

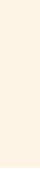

Aborde temprano la coagulopatía clínicamente significativa identificando la fuente $y / 0$ el defecto de coagulación 
Tabla 1. Recomendaciones del Patient Blood Management (PBM) en pandemia (continuación)

PBM para el manejo de la sangre de pacientes (del grupo de trabajo IFPBM-SABM)

\begin{tabular}{|l|l|l}
\hline Herramientas & Deficiencia de hierro y anemia & Pérdida sanguínea y sangrado \\
\hline $\begin{array}{l}\text { 6rincipios } \\
\text { generales (A) }\end{array}$ & - Toma de muestra y embalaje \\
& - Embolización radiológica \\
intervencionista & - Estrategia de transfusión restrictiva \\
& (reducir volumen de transfusión, \\
& cumplir con umbrales restrictivos de \\
& transfusión) \\
& - Esté atento a signos de sangrado \\
& postoperatorio \\
& - Monitorear durante el retiro/puente/ \\
& reanudación de ACOD y agentes \\
& antiplaquetarios \\
& - Prevenir el sangrado gastrointestinal \\
& (alimentación enteral, agentes \\
& reductores de ácido gástrico) \\
& - Evite y trate las infecciones con prontitud
\end{tabular}

6. Principios generales $(\mathrm{B})$

7. POS y pautas de procedimientos

- Identificar pacientes y procedimientos quirúrgicos con mayor riesgo de pérdida de sangre, anemia y coagulopatía.

- Remitir a los pacientes de alto riesgo inmediatamente al programa PBM

- Planificación quirúrgica preoperatoria para minimizar la extensión y el tiempo de la cirugía, incluida la embolización preoperatoria o las técnicas no invasivas

- Posponer o cancelar la cirugía electiva para dar tiempo a optimizar la recuperación hematológica

POS para detección, evaluación y manejo de anemia y deficiencia de hierro para entornos específicos:

- Precirugía y poscirugía

- Cáncer

- Insuficiencia cardiaca

- Enfermedad renal crónica

- Embarazo y posparto

- Pediatría

- Anemia adquirida en el hospital

- Pacientes con eritropoyesis con restricción de hierro

- Anemia por respuesta inflamatoria

- Manejo de agentes anticoagulantes

y antiplaquetarios antes de las

intervenciones

- Buscar historia de sangrado

- Algoritmos de manejo de sangrado

- Guía de procedimiento para el rescate celular

- Guía de procedimiento para HNA

- Mantener la normotermia

- Protocolo de hemorragia mayor

- Pautas sobre hierro oral vs. hierro intravenoso, preparaciones de hierro y dosificación

- Establecer «políticas para la transfusión de una sola unidad"

8. Sistema de - Toma de decisiones centrada en el paciente y basada en datos recopilación de - Mida el cambio con respecto a los resultados del paciente/ahorro de costos datos, evaluación - Reporte de cambios

comparativa y presentación de informes

9. Educación y formación continua

- Programas multidisciplinarios y multiprofesionales organizados y dirigidos por líderes locales

- Actualización periódica del currículo/contenido de aprendizaje (competencias)

- Asegurar cursos introductorios para el personal nuevo

10. Educación del paciente, información y consentimiento

- Desarrollar un plan de gestión educativa simplificado

- Establecer procedimientos para comunicarse con el plan de retratamiento de pacientes, riesgos/beneficios y obtener el consentimiento

- Comunicar el plan a todos los miembros del equipo

11. Infraestructura - Nombrar al personal de PBM y asignar/reasignar fondos en consecuencia

- Crear descripciones de trabajo para el personal dedicado de PBM

- Instale los dispositivos y equipos médicos necesarios

- Vuelva a diseñar las vías clínicas y la infraestructura para permitir la evaluación adecuada del paciente preoperatorio/preintervención y optimización

- Garantizar y asegurar zonas de espera y áreas de tratamiento apropiadas, particularmente para la optimización del paciente preoperatorio/preintervención

- Formar un comité multidisciplinario de PBM

IFPBM-SABM: Fundación Internacional para el Manejo de la Sangre del Paciente-Sociedad para el Avance del Manejo de la Sangre; POS: procedimientos operativos estandarizados; EPO: estándares de procedimientos operativos; PBM: manejo de la sangre de pacientes; ACOD: anticoagulantes orales directos; CCP: concentrado de complejo protrombínico; HNA: hemodilución normovolémica aguda; IBP: inhibidor de la bomba de protones; FIO2: fracción de oxígeno inspirado; COX: ciclooxigenasa; AINE: antiinflamatorio no esteroideo. Adaptada de Shander, et al., $2020^{31}$ 


\section{Recomendaciones del diagnóstico y tratamiento de la anemia en pacientes con cirugía electiva en espera}

Durante la pandemia de COVID-19, la actividad médica electiva se reduce notablemente y la cirugía electiva se pospone frecuentemente, dando prioridad a la cirugía urgente y de emergencia.

Las clínicas que manejan a estos pacientes funcionan para detectar, diagnosticar y tratar la deficiencia de hierro y otras causas de anemia durante la pandemia se encuentran cerradas y las citas canceladas; los análisis de sangre, resultados y las herramientas de diagnóstico están menos disponibles ahora, obstaculizando el diagnóstico y el tratamiento de la anemia y sus diversas causas.

Dar el tratamiento con hierro oral es la mejor manera de corregir esta anemia y mejorar las condiciones del paciente. Cuando la cirugía no sea urgente se debe retrasar para evitar la transfusión innecesaria. Las nuevas fórmulas de hierro oral tienen mayor absorción y tolerabilidad gastrointestinal, que permiten utilizar el tratamiento en días alternos en lugar de dosis diarias, para mejorar la absorción y el cumplimiento del tratamiento. Esta línea de acción es practicable para personas que no pueden abandonar su hogar debido a las medidas de cuarentena ${ }^{22}$.

Una vez reprogramada la cirugía, realizar pruebas que permitan evaluar la recuperación de la anemia (Tabla 2).

\section{Recomendaciones del manejo específico de donantes durante la pandemia de COVID-19}

El ECDC, así como el Centro Nacional de Sangre (Centro Nazionale Sangue, CNS), órgano asesor técnico y científico del Ministerio de Salud en Italia sobre asuntos relacionados con la sangre y los productos sanguíneos, han emitido una serie de medidas destinadas a mantener los altos estándares de donación de sangre y seguridad sanguínea en Italia, uno de los países con mayor incidencia de bajas relacionadas con la COVID-19 en todo el mundo ${ }^{10}$. Dentro de las medidas recomendadas se encuentran:

- Fortalecer las medidas de vigilancia de individuos que han estado en contacto con personas que han presentado una infección documentada por SARS-CoV-2 ${ }^{11}$.

- Diferir las donaciones de sangre en donantes que regresan de cualquier territorio nacional o internacional con un aviso de salud de viaje durante 14 días, donantes que hayan estado expuestos al riesgo de adquirir la infección por contacto con personas con infección documentada por SARS-CoV-2 y donantes con antecedentes de infección por SARS-CoV-2 o con síntomas compatibles con infección producida por SARS-CoV-2 que sean convalecientes.

- Posterior a la donación solicitar a los donantes que informen a sus centros de recolección de sangre en caso de síntomas compatibles con la infección por SARS-Cov-2 o en el caso del diagnóstico confirmatorio de infección dentro de los 14 días posteriores a la donación.

- Promover la implementación de procesos de triaje simples durante la recepción de donantes en las unidades de recogida de sangre, con el objetivo de evitar la posible propagación del virus en las salas de espera. Esto incluye la medición de la temperatura corporal fuera del área de recolección; si el donante presenta temperatura corporal de $37.5^{\circ} \mathrm{C}$ o más se define como un criterio para el aplazamiento temporal del donante.

- Solicitar a todo el personal que trabaja en las unidades de recolección de sangre que cumpla escrupulosamente los protocolos diseñados para prevenir la propagación de infecciones respiratorias, incluyendo infección por SARS-CoV-2.

- Acciones adicionales dirigidas a la gestión de donantes de sangre voluntarios para garantizar la donación continua y segura de componentes sanguíneos incluyen el llamado oportuno para donar sangre cuando se prevea escasez.

- Realizar planificación cuidadosa con cronograma de donantes para evitar un número excesivo de donantes en los centros de recolección de sangre.

- Cumplimiento de las medidas de distanciamiento social, uso de equipos de protección personal adecuado y el complimiento meticuloso de las normas de higiene.

- Los gobiernos deben considerar los antecedentes de viajes para donar sangre considerándolos como esenciales; se debe proporcionar certificado al donante si debe viajar al centro de recolección.

La implementación y aplicación de estas recomendaciones emitidas por el CNS facilita el manejo más seguro de las donaciones de sangre.

En Italia se mantuvieron 50,000 unidades por semana, garantizando así la autosuficiencia y seguridad de los 
Tabla 2. Guías relacionadas con el manejo de sangre del paciente por especialidad

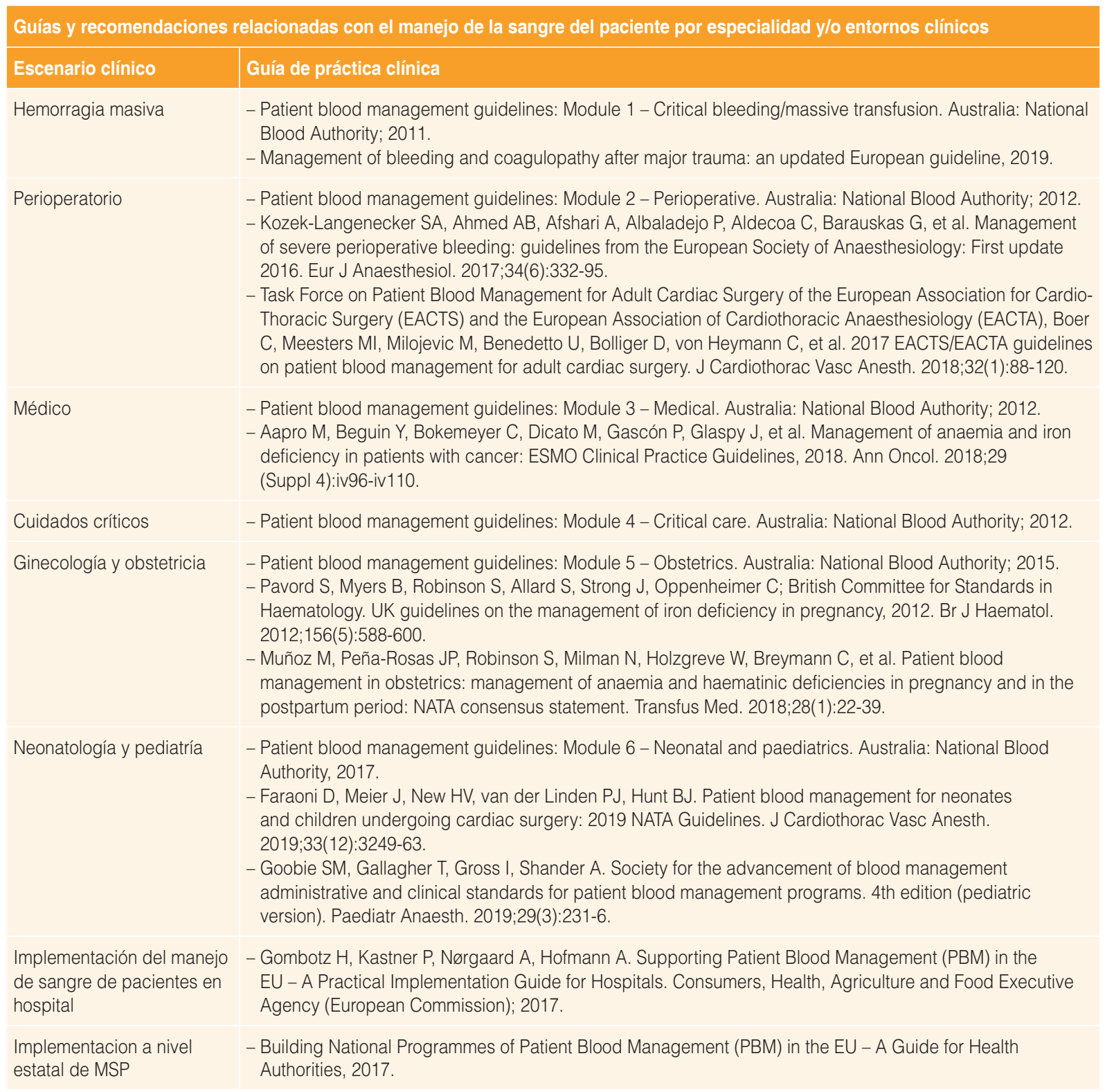

PBM: patient blood management.

Adaptada de Shander, et al., $2020^{31}$

componentes sanguíneos. En América Latina, en los países en desarrollo de transición como el nuestro este porcentaje de disminución llega a triplicarse, esto nos da idea de la escasez importante de las donaciones de sangre, con repercusiones serias en el abastecimiento y suministro de esta.

\section{Recomendaciones nacionales para el diferimiento de donantes de sangre}

En nuestro país la entidad reguladora en la recolección y abastecimiento de sangre es el Centro
Nacional de Transfusión Sanguínea (CNTS). En la actualización de lineamiento técnico para la selección y diferimiento de donantes en México, en relación con la COVID-19, emitido el 17 de abril del 2020, tomando en consideración las recomendaciones de la Organización Mundial de la Salud (OMS) y el marco del plan de acción para la sangre segura para la región de las Américas sobre el SARS y la seguridad de la sangre que son aplicables a virus emergentes como el coronavirus causante del síndrome respiratorio de Oriente Medio y el SARS-CoV-2, recomienda a los servicios de sangre lo siguiente: 
- Solicitar a los candidatos a donación evitar asistir a donar si presentan síntomas de infección respiratoria.

- Solicitar a los donantes que asistan al banco de sangre o centro de colecta en la medida posible sin acompañantes.

- Evitar aglomeraciones de candidatos a donación de sangre.

- Adecuar los mecanismos de selección de donantes (evaluación clínica e interrogatorio) considerando las siguientes pautas de diferimientos:

- Asintomático, sin contacto cercano. Se acepta para donación, siempre que en caso de realizar biometría hemática resulten los linfocitos mayores a $1,500 \mathrm{cel} . / \mu \mathrm{l}$.

- Asintomático, con contacto cercano. Diferimiento durante al menos 30 días.

- Sintomático, caso probable de COVID-19. Diferimiento por 30 días. Donación después de recuperación completa y conclusión de la terapia.

- Sintomático, caso sospechoso de COVID-19. Diferimiento por 30 días. Donación después de recuperación completa y conclusión de la terapia.

- Sintomático, COVID-19 excluido. Diferimiento por 30 días. Donación después de recuperación completa y conclusión de la terapia.

- Donantes viajeros de áreas con transmisión local reciente:

- Asintomático. Diferimiento durante al menos 30 días después del último día de regreso al país.

- Sintomático, caso probable COVID-19. Diferimiento por 30 días. Donación después de recuperación completa y conclusión de la terapia.

- Sintomático, caso sospechoso COVID-19. Diferimiento por 30 días. Donación después de recuperación completa y conclusión de la terapia.

- Sintomático, COVID-19 excluido. Diferimiento por 30 días. Donación después de recuperación completa y conclusión de la terapia.

- Donantes viajeros de áreas sin transmisión local reciente:

- Asintomático. Se acepta para donación, siempre que en caso de realizar biometría hemática resulten los linfocitos mayores a $1,500 \mathrm{cel} . / \mu \mathrm{l}$.
- Sintomático. Diferimiento durante al menos 30 días.

- Los servicios de sangre solicitarán a los donantes efectivos que informen si se diagnostican como caso probable o sospechoso de coronavirus dentro de 45 días después de la donación de sangre, o bien, si han sido diagnosticados con influenza dentro de 30 días después de la donación. En caso afirmativo, los servicios de sangre deberán retirar los productos sanguíneos aún no transfundidos y en caso de que ya fueran transfundidos notificar al receptor para su seguimiento epidemiológico y diagnóstico.

- Fortalecer la colecta de sangre con base en donaciones voluntarias habituales y no remuneradas.

- Comunicar y alentar al cumplimiento del personal de los servicios de sangre de las recomendaciones para los trabajadores de la salud sobre la prevención de la COVID-19.

- Mantener continuo contacto con las áreas de epidemiología para informarse acerca de la circulación del virus.

- Generar y comunicar planes de abastecimiento de sangre en situaciones de emergencias.

- Cuando se inicie la propagación del virus comunitaria se deberá favorecer la donación por medio del esquema de citas, ya sea vía telefónica o por internet, con el objetivo de disminuir la probabilidad de contagio de personas clínicamente sanas.

Hemos comparado los periodos de diferimientos establecidos por el CNTS, con respecto a los donantes y hay diferencia entre lo recomendado por el ECDC, pero cada país establece sus lineamientos considerando lo recomendado por la OMS.

\section{Consideraciones especiales}

En las emergencias y crisis sanitarias, las fuerzas de trabajo están sobrecargadas, las cadenas de suministro se ven interrumpidas, las capacidades de producción se reducen o se desvían y las infraestructuras se ven colapsadas ante el aumento de la demanda. Es importante considerar que la transfusión de CE se ha asociado con efectos clínicos negativos en el pulmón, como la lesión pulmonar aguda relacionada con la transfusión o la hipertensión pulmonar ${ }^{23}$. Estos efectos secundarios pueden no mejorar la gravedad del SDRA en pacientes afectados $^{24}$. Y producirán un consumo inadecuado de los componentes sanguíneos y disminución de la 
disponibilidad de estos, lo que conduce a la escasez de recursos médicos cruciales. Esta realidad se observa durante la pandemia de COVID-19 por la escasez de equipos de protección personal, ventiladores, camas de $\mathrm{UCl}$, medicamentos vitales y otros equipos críticos para trabajadores de la salud y personal de primera línea. Todo esto pone al personal médico en un gran riesgo personal y obliga a tomar decisiones difíciles con respecto a la asignación de los escasos recursos del paciente ${ }^{25}$. Teniendo en cuenta las numerosas incertidumbres de la pandemia de COVID-19, el alcance total de las consecuencias aún es desconocido.

Algunos modelos sugieren un costo humano directo en decenas de miles solo en los EE.UU., extrapolando a cientos de miles de muertes en todo el mundo. Indirectamente, a medida que las economías de todo el mundo sufren, las poblaciones enfrentan riesgos debido a la interrupción de los sistemas de atención médica y la reducción del acceso a la atención. Las medidas preventivas generan problemas de salud mental y pobreza. A menudo existe una relación inversa siniestra entre las medidas de prosperidad económica y varios indicadores de salud y bienestar. Existe una relación inversa siniestra entre las medidas de prosperidad económica y varios indicadores de salud y bienestar ${ }^{25}$.

El manejo de la sangre en los pacientes puede ejercer un papel importante en la disminución de la escasez a corto plazo en medio de la fase aguda de la pandemia, retomar todas estas medidas para las ondas posteriores hasta que se establezca la inmunidad del rebaño mediante una infección o vacunación generalizada y los desafíos de salud socioeconómica a largo plazo en el periodo posterior a la crisis de COVID-19 en el mundo. El enfoque principal del adecuado manejo de la sangre es necesario para mejorar los resultados de los pacientes, puede traducirse directamente en una menor carga de enfermedad y gravedad en pacientes individuales. Las prácticas de manejo del paciente previenen la anemia (un problema de salud global) y reducen las transfusiones de sangre alogénicas, lo que resulta no solo en la mejor asignación de recursos y ahorro de costos, sino también en la disminución de la morbilidad y mortalidad de los pacientes ${ }^{26}$.

Estos resultados mejorados y los ahorros de costos concomitantes se necesitan con urgencia a medida que lidiamos con la pandemia que aún no ha alcanzado su punto máximo, y los desafíos actuales siguen presentes.

Durante la pandemia de COVID-19, el aspecto más fundamental del manejo de la sangre del paciente es la prevención y el manejo de la anemia. Si bien el impacto exacto de la anemia en los resultados de los pacientes con COVID-19 aún no se comprende completamente, los datos muestran inequívocamente que el pronóstico de los pacientes con COVID-19 con afecciones preexistentes y crónicas es significativamente peor. Como tal, no es irracional esperar que la anemia también tenga un impacto negativo en los resultados de los pacientes con COVID-19; la prevención y el tratamiento generalizados de la anemia podrían conferir cierta protección contra los casos más graves de COVID-19.

El tipo de sangre puede contribuir a la susceptibilidad a la COVID-19. Los individuos con el grupo sanguíneo A pueden ser más vulnerables a la COVID-19, mientras que aquellos con el grupo sanguíneo $O$ pueden tener un riesgo significativamente menor de infección ${ }^{27}$. Sin embargo, aún se necesita más evidencia antes de poder confirmar esta observación.

Existen algunas preocupaciones prácticas para la seguridad del laboratorio para la transfusión de sangre durante la epidemia de COVID-19, que puede resolverse mediante la automatización parcial o total de las pruebas y equipos de desinfección adicionales, como uso de lámpara ultravioleta incorporada y la centrífuga de tarjeta con antiaerosol o desinfección en cada procedimiento.

Ante el brote de COVID-19, los departamentos de servicio de sangre deben garantizar una comunicación oportuna y precisa con los organismos reguladores e instituciones de salud pública, adoptar políticas flexibles, preparar planes de emergencia con antelación, asegurar que la recolección de sangre cumpla con las necesidades clínicas, mantener la seguridad del personal y los donantes de sangre, y minimizar la transmisión del virus por medio de la transfusión de sangre ${ }^{28}$.

La salud es la mayor de las bendiciones humanas y la humanidad unida es esencial para una comunidad global saludable ${ }^{29}$.

La epidemia de COVID-19 se superará mediante la concentración de las fuerzas sociales, la prevención, el control científico, la implementación precisa de políticas y una estrecha cooperación de toda la sociedad $^{30}$. 


\section{Conclusiones}

A medida que enfrentamos el mayor desafío de salud global de nuestras vidas, solo un esfuerzo de colaboración importante nos permitirá lograr un resultado positivo. Utilizando un enfoque centrado en el paciente, se deben aplicar principios probados basados en evidencias y continuar con los conceptos establecidos de consenso de expertos sobre buenas prácticas transfusionales.

El manejo de la sangre en todos los pacientes es una pieza del rompecabezas necesario para salvar la vida de los pacientes. Cada gota de sangre donada puede salvar y ser decisiva, aún más en la situación crítica que enfrentamos actualmente. El conocimiento de la COVID-19 evoluciona cada día.

Una estrategia de comunicación eficiente con los donantes de sangre voluntarios para motivarlos a realizar donaciones y la creación de redes de donantes, son actividades que se deben llevar a cabo. La información a los donantes sobre las medidas tomadas en cada centro de donación que garantizan su seguridad y medidas de protección para evitar contagio son esenciales para dar seguridad al donante.

Los bancos de sangre juegan un papel primordial para trabajar de manera cercana con los servicios de transfusiones que ayudarán a utilizar la terapia transfusional con indicaciones fundamentadas en el conocimiento científico: no transfundir para corregir resultados de laboratorio y no transfundir cuando un paciente tenga una opción alternativa de tratamiento que no sea un componente sanguíneo, razón por la que en esta revisión consideramos el manejo de la anemia por deficiencia de hierro. El utilizar de manera correcta las transfusiones mejora el suministro de sangre durante esta pandemia.

\section{Financiamiento}

No se recibió financiamiento ni patrocinio por ninguna organización o institución.

\section{Conflicto de intereses}

Los autores declaran no tener ningún conflicto de interés con el tema de la publicación.

\section{Responsabilidades éticas}

Protección de personas y animales. Los autores declaran que para esta investigación no se han realizado experimentos en seres humanos ni en animales.

Confidencialidad de los datos. Los autores declaran que han seguido los protocolos de su centro de trabajo sobre la publicación de datos de pacientes.

Derecho a la privacidad y consentimiento informado. Los autores declaran que en este artículo no aparecen datos de pacientes.

\section{Bibliografía}

1. COVID-19 Dashboard by the Center for Systems Science and Engineering (CSSE) at Johns Hopkins University (JHU) [Internet]. Johns Hopkins University. Disponible en: https://gisanddata.maps.arcgis.com/apps/opsdashboard/index.html\#/bda7594740fd40299423467b48e9ecf6

2. Backer JA, Klinkenberg D, Wallinga J. The incubation period of 2019 novel coronavirus (2019-nCoV) infections among travelers from Wuhan, China. Euro Surveill. 2020;25(5):2000062.

3. Fan BE, Chong VCL, Chan SSW, Lim GH, Lim KGE, Tan GB, et al. Hematologic parameters in patients with COVIDa19 infection. Am J Hematol. 2020;95(6):E131-E134.

4. Xiao F, Tang M, Zheng X, Liu Y, Li X, Shan H. Evidence for gastrointestinal infection of SARSCoV-2. Gastroenterology. 2020;158(6):1831-3.e3.

5. Fan BE, Ong KH, Chan SSW, Young BE, Chong VCL, Chen SPC, et al. Blood and blood product use during COVID-19 infection. Am J Hematol. 2020;95(7):E158-E160.

6. Gschwender AN, Gillard L. Disaster preparedness in the Blood Bank. Clin Lab Sci. 2017;30(4):250-7.

7. Shan $H$, Zhang P. Viral attacks on the blood supply: The impact of severe acute respiratory syndrome in Bejing. Transfusion. 2004:44:467-9.

8. Baron DM, Franchini M, Goobie SM, Javidroozi M, Klein AA, Lasocki S, et al. Patient blood management during the COVID-19 pandemic: a narrative review. Anaesthesia. 2020;75(8):1105-13.

9. Cho HJ, Koo JW, Roh SK, Kim YK, Suh JS, Moon JH, et al. COVID-19 transmission and blood transfusion: A case report. J Infect Public Health. 2020;13(11):1678-9.

10. Outbreak of acute respiratory syndrome associated with a novel coronavirus, Wuhan, China; first update [Internet]. European Centre for Disease Prevention and Control; 22 enero 2020 [consultado: 31/03/2020]. Disponible en: https://www.ecdc.europa.eu/sites/default/files/documents/ Risk-assessment-pneumonia-Wuhan-China-22-Jan-2020.pdf

11. Franchini M, Farrugia A, Velati C, Zanetti A, Romanò L, Grazzini G, et al. The impact of the SARS-CoV-2 outbreak on the safety and availability of blood transfusion in Italy. Vox Sang. 2020;115(8):603-5

12. Ranucci M, Baryshnikova E, Catelvecchio S, Pelissero G; Surgical and Clinical Outcome Research (SCORE) Group. Major bleeding, transfusions, and anemia: the deadly triad of cardiac surgery. Ann Thorac Surg. 2013;96(2):478-85.

13. Baron DM, Hochrieser H, Posch M, Metnitz B, Rhodes A, Moreno RP, et al. Preoperative anemia is associated with poor clinical outcome in non-cardiac surgery patients. Br J Anaesth. 2014;113(3):416-23.

14. Muñoz M, Acheson AG, Auerbach M, Besser M, Habler O, Kehlet $H$, et al. International consensus statement on the perioperative management of anemia and iron deficiency. Anaesthesia. 2017;72:233-47.

15. Blaudszun G, Muting KE, Butchart A, Gerrard C, Klein AA. The association between borderline preoperative anemia in women and outcomes after cardiac surgery: a cohort study. Anaesthesia. 2018;73:572-8.

16. Muñoz M, Laso-Morales MJ, Gómez-Ramírez, Cadellas M, Núñez-Matas MJ, García-Erce JA. Pre-operative haemoglobin levels and iron status in a large multicenter cohort of patients undergoing major elective surgery. Anaesthesia. 2017;72:826-34.

17. Rössler J, Schoenrath F, Seifert B, Kaserer A, Spahn GH, Falk V, et al. Iron deficiency in associated with higher mortality in patients undergoing cardiac surgery: a prospective study. Br J Anaesth. 2020;124(1):25-34.

18. Muñoz M, Gómez-Ramírez S, Cuenca J, García-Erce JA, Iglesias-Aparicio $\mathrm{D}$, Haman-Alcober $\mathrm{S}$, et al. Very-short-term perioperative intravenous iron administration and postoperative outcome in major orthopedic surgery: a pooled analysis of observational data from 2547 patients. Transfusion. 2014;54:289-99.

19. Camaschella C, Nai A, Silvestri L. Iron metabolism and iron disorders revisited in the hepcidin era. Haematologica. 2020;105:260-72.

20. Tardy AL, Pouteau E, Marquez D, Yilmaz C, Scholey A. Vitamins and minerals for energy, fatigue and cognition: a narrative review of the biochemical and evidence. Nutrients. 2020;12(1):228.

21. Stauder R, Valent $P$, Theurl I. Anemia at older age: etiologies, clinical implications, and management. Blood. 2018;131:505-14. 
22. Stoffel NU, Zeder C, Brittenham GM, Moretti D, Zimmermann MB. Iron absorption from supplements is greater with alternate day than with consecutive day dosing in iron-deficient anemic women. Haematologica. 2020;105(5):1232-9.

23. Baron DM, Lei C, Berra L. Old, older the oldest: red blood cell storage and the potential harm of using older red blood cell concentrates. Curr Opin Anaesthesiol. 2020;33:234-9.

24. Vlaar APJ, Toy P. Fung M, Looney MR, Juffermans NP, Bux J, et al. A consensus redefinition of transfusion-related acute lung injury. Transfusion. 2019;59:2465-76.

25. Rombolà G, Heidempergher M, Pedrini L, Farina M, Aucella F, Messa P et al. Practical indications for the prevention and management of SARSCoV-2 in ambulatory dialysis patients: lessons from the first phase of the epidemics in Lombardy. J Nephrol. 2020;33(2):193-6.

26. Meybohm P, Straub N, Füllenbach C, Judd L, Kleinerüschkamp A, Taeuber I, et al. Health economics of patient blood management: a cost-benefit analysis based on a meta-analysis. Vox Sang. 2020;115:182-8.

27. Zhao J, Yang Y, Huang H, Li D, Gu D, Lu X, et al. Relationship between the ABO blood group and the COVID-19 susceptibility. Clin Infect Dis. 2020 Aug 4:ciaa1150. doi: 10.1093/cid/ciaa1150. Online ahead of print.
28. Centro Nacional de la Transfusión Sanguínea 2020. Actualización del lineamiento técnico para la selección y diferimiento de donantes en México, con relación al coronavirus 2019-COVID-19 y otras infecciones respiratorias agudas [Internet]. México: Centro Nacional de la Transfusión Sanguínea. Disponible en: https://www.gob.mx/cnts/documentos/ recomendaciones-de-abordaje-para-atender-la-pandemia-de-virus-covid-19-en-los-servicios-de-sangre

29. Centros para el Control y la Prevención de Enfermedades. Nuevo coronavirus de 2019 (2019-nCoV), Wuhan, China. Resumen de la situación [Internet]. EE.UU.: Centros para el Control y la Prevención de Enfermedades [consultado: 02/03/2020]. Disponible en: https://www.cdc.gov/coronavirus/2019-nCoV/summary.html

30. Organización Mundial de la Salud. Nuevo coronavirus. Brotes y emergencias [Internet]. Organización Mundial de la Salud [consultado: 02/03/2020]. Disponible en: https://www.who.int/westernpacific/emergencies/novel-coronavirus

31. Shander A, Goobie SM, Warner MA, Aapro M, Bisbe E, Perez-Calatayud AA, et al. Essential role of patient blood management in a pandemic: a call for action. Anesth Analg. 2020;131(1):74-85. 